\title{
CORRECTION
}

\section{Correction to: Panel Discussion: Some Aspects of the Management of Patients with X-Linked Hypophosphataemic Rickets}

José-Vicente Torregrosa · Jaime Sánchez del Pozo • María Isabel Luiz Yanes •

Manuel Muñoz Torres

Published online: June 17, 2020

(C) Springer Healthcare Ltd., part of Springer Nature 2020

Correction to: Adv Ther (2020) 37:S121-S126

https://doi.org/10.1007/s12325-019-01208-w

In the original article, there is an error in age related reference range of Table 2 . The incorrect age range is given as $2-15$ months, the correct age range is $2-15$ years.

The original article can be found online at https://doi. org/10.1007/s12325-019-01208-w.

J.-V. Torregrosa ( $\square)$

Servicio de Nefrología y Trasplante Renal, Hospital Clinic, Universidad de Barcelona, Barcelona, Spain e-mail: vtorre@clinic.cat

J. Sánchez del Pozo

Sección de Endocrinología, Nutrición y

Dismorfología Pediátrica, Servicio de Pediatría,

Hospital Universitario 12 de Octubre, Madrid, Spain

M. I. Luiz Yanes

Unidad de Nefrología Pediátrica, Servicio de

Pediatría, Hospital Universitario Nuestra Señora de

Candelaria, Santa Cruz de Tenerife, Spain

M. Muñoz Torres

Departamento de Medicina, Universidad de Granada, UGC Endocrinología y Nutrición. Hospital Universitario San Cecilio, CIBERFES, Instituto de

Salud Carlos III, Instituto de Investigación

Biosanitaria de Granada, Granada, Spain 